\title{
Functional characterization of papain-like cysteine proteases genes in rice
}

\author{
Nino Marjohn ${ }^{1}$, Nogoy Franz M. ${ }^{1}$, Kim Me-Sun ${ }^{1}$, Ouk Sothea ${ }^{1}$, Yang Ju-Young ${ }^{1}$, \\ Lee Kye Dong ${ }^{1}$, Jung Yu Jin ${ }^{2}$, Kang Kwon Kyoo ${ }^{2}$, Cho Yong-Gu ${ }^{1 *}$ \\ ${ }^{1}$ Department of Crop Science, Chungbuk National University, Cheongju, Korea \\ ${ }^{2}$ Department of Horticultural Life Science, Hankyong National University, Ansung, Korea \\ *e-mail:ygcho@cbnu.ac.kr
}

Papain-like cysteine proteases (PLCP) are key enzymes involved in cell death as response to biotic stress. Functional genetic investigation of cysteine protease family members has been performed in a fragmentary scale to understand its specific role in plants. Highlights of research milestone for these proteases provide strong evidence on their diverse and overlapping roles in basal immunity and effector-triggered immunity. The objective of this study was to provide useful insights into biological function of three cysteine protease genes, $O_{s} C P 2, O s C P 3$, and $O s C P 5$, in rice. Overexpression of rice cysteine protease attenuated the virulence of Xanthomonas oryzae pv. oryzae race K3a in all transgenic lines which displayed moderate resistance as indicated by shorter lesion lengths (OsCP2ox, $6.82 \mathrm{~cm}$; OsCP3ox, $5.55 \mathrm{~cm}$; and OsCP5ox, $5.40 \mathrm{~cm}$ ) than wild type Dongjin $(16.07 \mathrm{~cm})$ whereas RNAi-mediated knockdown of $O s C P 3$ resulted in severe bacterial leaf blight symptoms $(17.1 \mathrm{~cm})$. Abiotic screening revealed the biological significance of these three cysteine protease genes, especially of $O s C P 3$, against salinity stress for which rice exhibited moderate tolerance ( salinity score $=5.0$ to 5.2 ). This study provides experimental evidence for roles of papain-like cysteine protease in improving resistance of rich against Xanthomonas oryzae pv. oryzae and tolerance against salinity stress, suggesting that these genes could be used as a valuable resource to be employed in rice breeding program to improve its ability to withstand biotic and abiotic stresses. Acknowledgements: This study was supported by the Russian Science Foundation. This work was supported by a grant from the Next-Generation BioGreen 21 Program (PJ01330201), RDA, Republic of Korea. 